\title{
Desenvolvimento de gelado comestível adicionado de linhaça (Linum usitatis- simum L.) e quinoa (Chenopodium quinoa Willd)
}

\begin{tabular}{ll}
\hline \multirow{3}{*}{ Catia Cristine Urnau Vivian } & $\begin{array}{l}\text { SEARA Alimentos Ltda, Três Passos -RS. } \\
\text { Universidade Estadual do Rio Grande do Sul, } \\
\text { Três Passos, RS, Brasil } \\
\text { E-mail: catia.vivian@seara.com.br }\end{array}$ \\
\hline \multirow{2}{*}{$\begin{array}{l}\text { Instituto Federal Farroupilha. Unidade de } \\
\text { Gislaine Hermanns }\end{array}$} & $\begin{array}{l}\text { Santo Augusto. Instituto Federal Farroupilha, } \\
\text { Santo Augusto, RS, Brasil } \\
\text { E-mail: daiane.preci@iffarroupilha.edu.br }\end{array}$ \\
& Email: gislaine.hermanns@iffarroupilha.edu.br \\
\hline Fernanda Hart Weber & $\begin{array}{l}\text { Universidade Estadual do Rio Grande do Sul. } \\
\text { Unidade de Três Passos. Universidade Esta- }\end{array}$ \\
& dual do Rio Grande do Sul, Três Passos, RS, \\
& Brasil \\
& E-mail: fernanda-hart@uergs.edu.br \\
\hline
\end{tabular}

Recebido em: 8 jun. 2017. Revisado: 16 ago. 2017. Aceito: 5 set. 2017.

DOl: http://dx.doi.org/10.21674/2448-0479.33.508-527

\section{Resumo}

A população está em busca pela melhoria da qualidade de vida, sendo esta realizada através do consumo de novos produtos com características funcionais, que possam ocasionar efeitos benéficos à saúde. Este trabalho teve como objetivo elaborar um gelado comesRev. Elet. Cient. UERGS, v. 3, n. 3, p. 508-527, 2017 
tível adicionado de linhaça e quinoa. Foram desenvolvidas cinco formulações de gelado comestível, sendo uma padrão sem adição de linhaça e quinoa, e nas demais formulações foram utilizadas diferentes concentrações desses ingredientes. A avaliação da aceitabilidade sensorial foi realizada por 100 julgadores não-treinados, estes avaliaram a aceitação global das formulações, utilizando o teste de escala hedônica de nove pontos. A amostra padrão (A) e a amostra $B, C$ e $E$, tiveram melhor aceitação, diferente da amostra $D$ que diferiu significativamente da amostra $A$ (padrão) nos atributos de sabor, textura, sabor residual e impressão global. Foram realizadas análises físico-químicas e microbiológicas na amostra $A$ e na amostra $E$ devido a mesma apresentar os dois ingredientes funcionais (linhaça e quinoa) e também devido a mesma não se diferenciar da amostra A, apresentando praticamente o mesmo nível de aceitabilidade pelos provadores. As análises microbiológicas de ambas as amostras estão de acordo com os padrões legais vigentes e as análises físicoquímicas apresentaram resultados satisfatórios. O gelado comestível da amostra $\mathrm{E}$ apresentou $1,1 \mathrm{~g}$ de fibra por $100 \mathrm{~g}$ de amostra, diferente da amostra $A$ que não apresentou concentração de fibra. Esse resultado permite concluir que a amostra $E$ possui ingredientes funcionais no desenvolvimento de produtos com concentração de fibras, sendo um produto diferenciado na alimentação.

Palavras-chave: Linhaça e Quinoa. Avaliação sensorial. Fibra alimentar. 


\section{Abstract \\ Development of edible ice cream with linseed (Linum usitatis-simum L.) and quinoa (Che- nopodium quinoa Willd)}

The population is looking for an improvement in the quality of life, which is achieved through the consumption of new products with functional characteristics that may have beneficial effects on health. This work had as a goal to elaborate an edible ice cream added of flaxseed and quinoa. Five formulations of edible ice cream were developed, a standard without addition of flaxseed and quinoa, and in the other formulations different concentrations of these ingredients were used. The evaluation of sensory acceptability was performed by 100 untrained judges, who evaluated the overall acceptance of the formulations using the nine-point hedonic scale test. The standard sample (A) and sample B, C and E had a better acceptability, different from sample $D$, which differed significantly from sample $A$ (standard) on the taste, texture, residual taste and overall impression attributes. Physicochemical and microbiological analyzes were carried out in sample $A$ and sample $E$ because it presented the two functional ingredients (flax and quinoa) and also because it did not differentiate from sample $A$, presenting practically the same level of Acceptability by tasters. The microbiological analyzes of both samples are in accordance with the current legal standards and the physicochemical analyzes presented satisfactory results. The edible ice cream of sample $\mathrm{E}$ presented $1.1 \mathrm{~g}$ of fiber per $100 \mathrm{~g}$ of sample, different from sample $A$ that did not present fiber concentration. This result allows to conclude that sample $E$ has functional ingredients in the development of products with fiber concentration, being a differentiated product in the feed.

Keywords: Functional. Sensory evaluation. Dietary fiber. 


\section{Introdução}

No decorrer dos anos, com o avanço tecnológico no setor de produtos alimentícios a população vem promovendo mudanças nos conceitos de nutrição, modificando seus hábitos alimentares através de produtos mais saudáveis e que tragam benefícios à saúde. Dentre eles, estão os alimentos funcionais que vêm conquistando o mercado pelos seus efeitos benéficos para a saúde humana, atendendo o binômio "alimentação e saúde" (SOUZA et al., 2003).

Acompanhando as exigências colocadas pela transformação do estilo de vida da população, a ciência dos alimentos vem possibilitando a inovação em produtos alimentícios, como forma de atender à busca das pessoas por alimentos mais saudáveis, com benefícios à saúde ou que tenham alguma propriedade funcional (NASCIMENTO et al., 2012). Segundo Raud (2008), os alimentos funcionais estão se transformando em uma nova fronteira do mercado de alimentos, com grandes possibilidades de crescimento.

$O$ ingrediente funcional que vem sendo utilizado na alimentação são as fibras devido a sua função na redução dos níveis de colesterol sanguíneo e diminuição dos riscos de desenvolvimento de câncer, devido apresentar capacidade de retenção de substâncias tóxicas ingeridas, redução do tempo do trânsito intestinal, promovendo melhor eliminação do bolo fecal e formação de substâncias protetoras pela fermentação bacteriana dos compostos de alimentação (ANJO, 2004). Devido essas funções, as fibras são muito importantes na alimentação diária, principalmente na alimentação do público infantil.

Dentre os alimentos ricos em fibras, destaca-se a linhaça, que é classificada como alimento funcional, por possuir a principal 
fonte vegetal de ômega 3 e ômega 6 (ácidos-graxos poliinsaturados) (GALVÃO et al, 2008), que regulam o colesterol e a pressão arterial (MARQUES, 2008), além de possuir ação antioxidante, por conter compostos fenólicos. A linhaça é caracterizada como um alimento rico em fibra, possibilitando muitos benefícios à saúde (TRUCOM, 2006).

Outro ingrediente funcional de grande importância é a quinoa, que possui alto valor nutritivo, sendo considerado um grão alternativo na alimentação humana. A semente de quinoa é rica em aminoácidos essenciais, além de possuir alto valor proteico e várias vitaminais (BRAGA; MENDONÇA, 2010). O mineral ferro encontrase presente em boa quantidade na semente de quinoa, numa eficiência elevada, podendo superar o sulfato ferroso, que é administrado para a obtenção de ferro, sendo essa uma das características pela qual a mesma é classificada como alimento funcional (SPEHAR, 2006).

Os produtos lácteos processados com ingredientes funcionais possuem efeitos benéficos à alimentação humana, como por exemplo, os iogurtes com probióticos que melhoram a saúde intestinal, os leites enriquecidos com ferro que ajudam na prevenção e no tratamento da anemia, ou os leites enriquecidos com vitaminas e com o ácido ômega-3 que ajudam no controle do colesterol (RAUD, 2008). Desta forma, a produção de "gelado comestível" adicionado de ingredientes funcionais se constitui numa das alternativas que vem sendo utilizada pelas indústrias de alimentos, possibilitando aos seus consumidores uma nova opção alimentar. Além disso, o gelado comestível é um produto muito apreciado pelas crianças, sendo de grande importância o desenvolvimento deste produto adicionado de ingredientes funcionais. 
Este trabalho teve o objetivo de desenvolver um gelado comestível adicionado de linhaça e quinoa com caraterísticas físicoquímicas, microbiológicas e sensoriais satisfatórias, visando atender as expectativas de consumidores em relação a saúde, nutrição, funcionalidade e consumo de um alimento seguro.

\section{Materiais e Métodos}

O gelado comestível adicionado de linhaça e quinoa foi produzido em uma Indústria de Gelados Comestíveis da Região Celeiro - RS e as análises microbiológicas e físico-químicas foram realizadas nos Laboratórios de Microbiologia e Bromatologia, do Instituto Federal de Educação, Ciência e Tecnologia Farroupilha de Santo Augusto - RS, sendo que a análise de sódio foi realizada pela Universidade Regional do Noroeste do Estado do Rio Grande do Sul (UNIJUI). A análise de fibra alimentar foi realizada pelo CEPA (Centro de Pesquisa em Alimentação da Universidade de Passo Fundo).

Os ingredientes utilizados para a elaboração do gelado comestível funcional foram: leite, sacarose (açúcar refinado), glucose, cacau em pó, emulsificante e estabilizante, linhaça em pó dourada estabilizada e farinha de quinoa.

O processo de fabricação do gelado comestível consistiu nas seguintes etapas: pesagem dos ingredientes, preparação da calda, pasteurização, homogeneização, resfriamento, maturação, congelamento e incorporação de ar, envase e endurecimento.

Para a fabricação do gelado comestível primeiramente foi realizada a adição do leite no pasteurizador (MDG, Modelo PG 150L, São Carlos - SP, Brasil) e em seguida realizou-se a pesagem Rev. Elet. Cient. UERGS, v. 3, n. 3, p 508-527, 2017 
dos ingredientes secos (açúcar, leite em pó e glicose) que foram adicionados ao leite com uma temperatura de $25^{\circ} \mathrm{C}$, para a obtenção da calda. Para a produção do gelado comestível foi realizada a pasteurização rápida da calda, que consiste em atingir a temperatura de 72 a $75^{\circ} \mathrm{C}$ por 15 segundos, em seguida a calda foi resfriada até $4^{\circ} \mathrm{C}$.

Após o processo da pasteurização, a calda foi transferida para a tina de maturação (MDG, Modelo TM 150L, São Carlos - SP, Brasil), onde permanece de 4 a 24 horas com temperatura de $4^{\circ} \mathrm{C}$. Passado o tempo mínimo de maturação, a calda foi utilizada para a fabricação do gelado comestível, onde realizou-se a medida desejada de calda, colocando-a diretamente no liquidificador industrial (MDG, Modelo MI 180, São Carlos - SP, Brasil), sendo também adicionado o sabor de cacau e o emulsificante, deixando bater por cerca de 20 segundos. Após foi adicionada a quantidade de linhaça e/ou quinoa desejada na calda, sendo encaminhada para a sorveteira descontínua (MDG, Modelo 80/100, São Carlos - SP, Brasil), onde a mistura sofreu $\mathrm{o}$ processo de batedura e congelamento. Após 10 minutos retirou-se o gelado comestível, que foi acondicionado em recipiente apropriado e armazenado em freezer $\mathrm{a}-18^{\circ} \mathrm{C}$.

Para a fabricação do gelado comestível foram processadas cinco formulações, partindo-se de uma formulação básica ou padrão. A primeira formulação foi desenvolvida no sabor de cacau em pó sem adição de linhaça em pó dourada e quinoa. As outras quatro foram balanceadas com adição de linhaça em pó dourada e quinoa em diferentes proporções, conforme demonstrado na Tabela 1. 
Tabela 1 - Gelado comestível com diferentes concentrações de ingredientes funcionais.

\begin{tabular}{cccc}
\hline $\begin{array}{c}\text { Identificação da } \\
\text { amostra }\end{array}$ & Sabor & Linhaça & Quinoa \\
\hline A & Cacau & - & - \\
B & Cacau & $10 \%$ & - \\
C & Cacau & - & $10 \%$ \\
D & Cacau & $10 \%$ & $10 \%$ \\
E & Cacau & $5 \%$ & $5 \%$ \\
\hline
\end{tabular}

Após a elaboração das cinco formulações, estas foram avaliadas sensorialmente utilizando o teste de aceitação, com uso de Escala Hedônica de nove pontos. Para essa análise os julgadores receberam cinco amostras de gelado comestível, sendo que os mesmos foram orientados a provar cada uma das amostras, avaliando as características de cor, sabor, textura, impressão global e sabor residual, expressando o seu grau de gostar ou desgostar do produto. O teste de escala hedônica utilizou-se de nove pontos com categorias variando de 9-gostei muitíssimo, 8-gostei muito, 7-gostei moderadamente, 6-gostei ligeiramente, 5-indiferente, 4- desgostei ligeiramente, 3-desgostei moderadamente, 2-desgostei muito, 1desgostei muitíssimo. A avaliação sensorial contou com a colaboração de 100 julgadores não treinados, selecionados de maneira aleatória.

Para a análise sensorial o projeto foi submetido para avaliação pelo Comitê de Ética - Plataforma Brasil sob o número do parecer 971.512. Os participantes do teste de aceitação receberam o Termo de Consentimento Livre e Esclarecido (T.C.L.E), aprovado quanto aos aspectos éticos da pesquisa.

Os dados referentes à aceitação das cinco amostras foram primeiramente submetidos a uma análise de variância (ANOVA) e as 
médias comparadas através do teste de Tukey, tendo-se como fontes de variação amostras e provadores. A formulação com níveis de aceitabilidade mais elevada, ou seja, caracterizada como mais aceita, foi conduzida para a realização das análises físico-químicas e microbiológicas, juntamente com a amostra padrão.

As análises físico-químicas de composição centesimal dos gelados comestíveis foram realizadas em triplicatas (umidade, proteína, lipídeos, cinzas, carboidratos, fibra alimentar e sódio), sendo realizadas segundo o Instituto Adolfo Lutz (2004).

As análises microbiológicas obrigatórias para a avaliação das condições higiênicos-sanitárias de fabricação de gelados comestíveis como Staphylococcus coagulase positiva, Coliformes a $45^{\circ} \mathrm{C}$ e Salmonella spp, foram realizadas de acordo com a Instrução Normativa $\mathrm{N}^{\circ} 62$, DE 2003, do MAPA (BRASIL, 2003), e os padrões avaliados conforme a RDC o 12 de 2 de janeiro de 2001, da Anvisa (BRASIL, 2001).

\section{Resultados e Discussão}

Os gelados comestíveis produzidos com adição de linhaça e quinoa foram submetidos à análise sensorial com provadores nãotreinados, os quais avaliaram o produto através da escala hedônica de 9 pontos quanto ao sabor, cor, textura, impressão global e sabor residual.

Na Tabela 2 estão apresentados os resultados dos atributos sensoriais das cinco formulações submetidas à avaliação sensorial. A partir dos resultados da análise sensorial, constatou-se que em todos os atributos avaliados, a amostra D apresentou-se com as 
menores médias encontrando-se na escala entre 6 e 7 (gostei ligeiramente e gostei moderadamente), apresentando diferença estatística nos atributos sabor, textura, sabor residual e impressão global da amostra A (padrão). Somente na avaliação da cor do produto a amostra $D$ não diferiu estatisticamente da amostra $A$ (padrão), demonstrando a menor aceitabilidade dentre as amostras avaliadas.

Para a amostra $E$ verificou-se que esta apresentou as maiores médias para os atributos cor e textura se comparada com a amostra A (padrão), porém sem diferir estatisticamente.

Tabela 2 - Médias e desvios-padrão dos atributos sensoriais das cinco formulações de gelado comestível.

\begin{tabular}{cccccc}
\hline $\begin{array}{c}\text { Identificação } \\
\text { da Amostra }\end{array}$ & Cor & Sabor & Textura & $\begin{array}{c}\text { Sabor } \\
\text { Residual }\end{array}$ & $\begin{array}{c}\text { Impressão } \\
\text { Global }\end{array}$ \\
\hline Amostra (A) & $7,45 \mathrm{ab}$ & $7,58 \mathrm{a}$ & $7,13 \mathrm{a}$ & $7,40 \mathrm{a}$ & $7,67 \mathrm{a}$ \\
& $\pm 1,28$ & $\pm 1,40$ & $\pm 1,55$ & $\pm 1,34$ & $\pm 1,09$ \\
Amostra (B) & $7,41 \mathrm{~b}$ & $7,35 \mathrm{a}$ & $7,01 \mathrm{ab}$ & $6,95 \mathrm{ab}$ & $7,14 \mathrm{~b}$ \\
& $\pm 1,35$ & $\pm 1,65$ & $\pm 1,53$ & $\pm 1,73$ & $\pm 1,72$ \\
Amostra (C) & $7,51 \mathrm{ab}$ & $7,15 \mathrm{a}$ & $7,18 \mathrm{a}$ & $7,01 \mathrm{a}$ & $7,26 \mathrm{ab}$ \\
& $\pm 1,10$ & $\pm 1,48$ & $\pm 1,51$ & $\pm 1,45$ & $\pm 1,35$ \\
Amostra (D) & $7,38 \mathrm{~b}$ & $7,02 \mathrm{~b}$ & $6,52 \mathrm{~b}$ & $6,52 \mathrm{~b}$ & $7,03 \mathrm{~b}$ \\
& $\pm 1,34$ & $\pm 1,47$ & $\pm 1,69$ & $\pm 1,73$ & $\pm 1,58$ \\
Amostra (E) & $7,78 \mathrm{a}$ & $7,40 \mathrm{a}$ & $7,34 \mathrm{a}$ & $7,08 \mathrm{a}$ & $7,42 \mathrm{ab}$ \\
& $\pm 1,07$ & $\pm 1,34$ & $\pm 1,55$ & $\pm 1,54$ & $\pm 1,43$ \\
\hline
\end{tabular}

Médias com letras diferentes, em uma mesma coluna, diferem entre si, pelo teste de Tukey ao nível de 5\% de significância.

A partir dos dados da avaliação sensorial (Tabela 2), verificou-se que, entre as características analisadas de cor, todas as amostras se enquadram se enquadraram com médias acima de 7 (equivalente a gostei moderadamente), não se diferenciando da amostra A (padrão). A amostra A (padrão) e a amostra $E$ apresentaram as maiores médias nos atributos de sabor, sabor residual e impressão global. A amostra D diferiu significativamente da amostra A (padrão) nos atributos de sabor, textura, sabor residual e impressão global, devido possuir maior concentração de ingredientes fun- 
cionais na amostra, ocasionando a modificação sensorial desses atributos.

Para a realização das análises físico-químicas e microbiológicas foi escolhida a amostra $\mathrm{E}$ devido a mesma apresentar os dois ingredientes funcionais (linhaça e quinoa) e também devido a mesma não se diferenciar da amostra $A$ (padrão), apresentando praticamente o mesmo nível de aceitabilidade pelos provadores em ambas as amostras. Diferente da amostra $D$ que se diferenciou em alguns aspectos da amostra A (padrão), apresentando menores médias de aceitação pelos provadores, apresentando os dois ingredientes funcionais na maior porcentagem aplicada na formulação do gelado comestível.

A Tabela 3 apresenta os resultados das análises microbiológicas realizadas nas formulações $A$ e $E$.

Tabela 3 - Análises microbiológicas dos gelados comestíveis das formulações $\mathrm{Ae} E$

\begin{tabular}{cccc}
\hline Amostras & $\begin{array}{c}\text { Coliformes a 45으. } \\
(\mathrm{NMP} / \mathrm{g})\end{array}$ & $\begin{array}{c}\text { Estafilococos } \\
\text { coagulase } \\
\text { positiva }\end{array}$ & Salmonella spp. \\
\hline Padrão RDC N $\mathrm{N}^{\circ} 12^{*}$ & $5 \times 10$ & $5 \times 10^{2}$ & Ausente \\
$\mathrm{A}^{* *}$ & $\mathrm{ND}$ & $\mathrm{ND}$ & Ausente \\
$\mathrm{E}^{\star * *}$ & $\mathrm{ND}$ & $\mathrm{ND}$ & Ausente \\
\hline
\end{tabular}

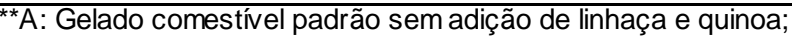

${ }^{* * *} \mathrm{E}$ : Gelado comestível com adição de $5 \%$ de linhaça e $5 \%$ de quinoa;

*Tolerância Máxima para amostra indicativa;

ND: Não Detectado.

Os resultados microbiológicos apresentados estão de acordo com o estabelecido pela Resolução RDC №12, de 02 de janeiro de 2001 da Agência Nacional de Vigilância Sanitária - ANVISA (BRASIL, 2001), demonstrando que o gelado comestível encontra- 
se em perfeitas condições de consumo, não ocasionando riscos para o consumidor.

A Tabela 4 apresenta os resultados das análises físicoquímicas realizadas nas duas formulações ( $A$ e E).

Tabela 4 - Análises físico-químicas dos gelados comestíveis das formulações $A$ e E.

\begin{tabular}{|c|c|c|}
\hline Componentes & A & $E$ \\
\hline Umidade $^{1}(\%)$ & $75,46 \pm 0,18$ & $64,48 \pm 0,22$ \\
\hline Proteína1 (\%) & $5,37 \pm 0,41$ & $5,43 \pm 0,31$ \\
\hline Gorduras Totais ${ }^{1}(\%)$ & $0,7 \pm 0,1$ & $1,16 \pm 0,06$ \\
\hline Gorduras Trans ${ }^{1}(\%)$ & ND & ND \\
\hline Cinzas $^{1}(\%)$ & $4,37 \pm 0,03$ & $4,35 \pm 0,20$ \\
\hline Carboidratos $^{2}(\%)$ & $14,01 \pm 0,49$ & $24,58 \pm 0,36$ \\
\hline Fibra Alimentar ${ }^{1}(\%)$ & $0 \pm 0$ & $1,1 \pm 0,01$ \\
\hline Valor Calórico* (Kcal/100g) & 87,08 & 128,76 \\
\hline Sódio** (mg/L) & $74,27 \pm 0,80$ & $86,53 \pm 3,35$ \\
\hline $\begin{array}{l}1 \text { Média aritmética de três deter } \\
2 \text { Calculado por diferença. } \\
\text { * Valor calórico calculado por } \\
{ }^{* *} \text { Para Sódio a concentração } \\
\text { ND: Não Detectado. }\end{array}$ & $\begin{array}{l}\text { ações } \pm \text { desvio } \\
\text { g de amostra. } \\
\text { mg/l. }\end{array}$ & \\
\hline
\end{tabular}

O gelado comestível da amostra A (padrão) apresentou maior teor de umidade com $75,67 \%$ do que o gelado comestível adicionado de fibra (amostra E) com 64,67\% de umidade (Tabela 3). Esta variação deve-se à adição de linhaça e quinoa na formulação do gelado comestível aumentando o teor de sólidos solúveis, resultando em um produto mais consistente. Conforme a RDC no 266 de 22 de setembro de 2005, da Agência Nacional de Vigilância Sanitária - ANVISA (BRASIL, 2005), que fixa o padrão de identidade e qualidade de gelados comestíveis, preparados, pós para o preparo e Rev. Elet. Cient. UERGS, v. 3, n. 3, p 508-527, 2017 
bases para gelados comestíveis, não existem valores específicos para umidade em sorvetes.

Em relação ao teor de proteína não houve diferença entre as duas formulações de gelado comestível, os resultados de ambas amostras podem ser considerados semelhantes, com alto valor proteico, atendendo a especificação mínima de 2,5\%. Segundo Silva e Lannes (2011), os gelados comestíveis fabricados com misturas de açúcar e tipos diferentes de gordura não apresentam variações no conteúdo de proteína.

O gelado comestível da amostra A (padrão), apresentou teor de $0,8 \%$ de lipídeos, diferente da amostra $E$ que apresentou um teor de $1,2 \%$, devido a adição de linhaça que é rica em ácidos graxos insaturados, benéficos à saúde. Segundo Marshall e Arbuckle (1996), a gordura láctea é o ingrediente de maior importância no sorvete e pode variar de 0 a $24 \%$. Desta forma, pode se caracterizar ambos os sorvetes com teores reduzidos de gordura, contribuindo para a dieta alimentar, pois o consumo de dietas ricas em gordura está intimamente ligado com o desenvolvimento de doenças crônicas, como a obesidade e doenças cardiovasculares (NEUTZLING et al., 2007).

De acordo com Madrid et al. (1996), os sorvetes são ricos em minerais como cálcio, sódio, potássio, magnésio entre outros, devido apresentar nas suas formulações leite. Neste estudo o teor de cinzas da amostra $A$ (padrão) com a amostra $E$, não apresentaram diferenças entre as formulações, sendo que os teores de cinzas encontrados mostram que ambas as amostras possuem quantidade considerável de minerais em sua composição.

$O$ valor de sódio encontrado na amostra $A$ (padrão), foi menor com 74,27mg/l do que a amostra E com 86,53mg/l (Tabela 3). Conforme o Informe técnico N. 50/2012, da Agência Nacional de 
Vigilância Sanitária - ANVISA (BRASIL, 2012), ambas as amostras podem ser caracterizadas com conteúdo baixo de sódio, porque apresentam concentração menor de $120 \mathrm{mg}$ de sódio por $100 \mathrm{~g}$ ou $\mathrm{mL}$.

Devido à adição dos ingredientes funcionais houve um aumento no teor de fibra alimentar da amostra $E$ em relação a amostra A (Tabela 3). A amostra $E$ apresentou a concentração de $1,1 \mathrm{~g}$ de fibra alimentar para $100 \mathrm{~g}$ de gelado comestível, enquanto a amostra A não possui fibra alimentar. Esse resultado se deve a adição dos ingredientes funcionais que são considerados importantes fontes de fibra alimentar.

Boff et al. (2013), desenvolveram um sorvete de chocolate utilizando fibra de casca de laranja como substituto de gordura, a fibra obtida nos dois sorvetes elaborados variou de acordo com a quantidade adicionada, na formulação $F 1$ - foram obtidos $0,21 \mathrm{~g}$ de fibras por $100 \mathrm{~g}$ de sorvete, e na formulação F2 - foram obtidos $0,31 \mathrm{~g}$ de fibras por $100 \mathrm{~g}$ de sorvete. Isso demonstra que a concentração de fibra de linhaça e quinoa adicionada ao gelado comestível foi superior, elevando o valor nutricional do produto.

A fibra alimentar quantificada na amostra $E$ pode auxiliar na dieta alimentar do ser humano, principalmente para o público infantil, pelo fato de possuir sabor de cacau sendo este considerado um sabor aceitável e muitas vezes o preferido pelas crianças, além de possuir ingredientes que contribuem para as necessidades nutricionais diárias da alimentação. Segundo, Mello et al (2004), deve-se encorajar a ingestão de fibras pelas crianças e desestimular o consumo de alimentos ricos em colesterol e gordura saturada, bem como o uso excessivo de sal e açúcar refinado.

O consumo de fibras na alimentação proporciona diversos benefícios a saúde humana. Segundo Nevez e Ribeiro (2003), as Rev. Elet. Cient. UERGS, v. 3, n. 3, p 508-527, 2017 
fibras reduzem a taxa glicêmica em diabéticos, atuando na prevenção de câncer de cólon, através da diminuição da presença de substâncias tóxicas na luz intestinal e pela maior eliminação fecal, além disso, contribuem para constipação intestinal crônica e no controle dos níveis de colesterol séricos.

De acordo com Santos (2006), em 100g de grão de linhaça, em média $30 \mathrm{~g}$ são fibras alimentares, divididas em insolúveis e solúveis. As fibras insolúveis ocasionam melhoras no sistema digestivo, aumentando o bolo fecal e à redução do período de trânsito intestinal. Já a fração de fibra solúvel, auxilia na manutenção dos níveis de glicose no sangue e redução dos níveis de colesterol sanguíneo (MORRIS, 2007).

A linhaça pode ser considerada um alimento funcional devido ao fato de produzir efeitos benéficos a saúde. De acordo Chinelate et al. (2012), a linhaça é um alimento vegetal único que oferece benefícios potenciais para a saúde cardiovascular por ser fonte importante de ácido $\alpha$-linolênico (ômega 3) e de lignanas. Além disso, é importante para o controle de diabetes, determinados tipos de câncer e na redução de processos inflamatórios.

A quinoa apresenta benefícios para a alimentação, segundo Alves et al. (2008), é um alimento de elevada qualidade protéica, possuindo todos os aminoácidos essenciais, para a nutrição do organismo humano, podendo ser considerada uma proteína de excelente qualidade (SILVA et. al., 2010). A semente de quinoa também é rica em uma série de vitaminais e é uma importante fonte de ácidos graxos poliinsaturados, que auxiliam no controle de doenças cardiovasculares (BRAGA; MENDONÇA, 2010). 


\section{Considerações finais}

De acordo com os resultados da avaliação sensorial, é possível desenvolver um produto com ingredientes funcionais, com características sensoriais aceitáveis pelo mercado consumidor, pois a avaliação sensorial mostrou uma boa aceitação do gelado comestível adicionado de linhaça e quinoa na proporção de $5 \%$ cada.

A adição de linhaça e quinoa promoveram um incremento no teor de fibra alimentar, tornando o gelado comestível estudado (amostra E), um produto com maior teor de fibra alimentar, auxiliando na dieta alimentar principalmente do público infantil.

Além disso, a amostra contendo os ingredientes funcionais não diferiu sensorialmente em nenhum atributo comparado com a amostra padrão. Esse resultado permite concluir que o gelado comestível adicionado de linhaça e quinoa é um produto diferenciado, com aceitação sensorial semelhante ao tradicional, porém com elevado valor nutricional.

\section{Referências}

ALVES, L. F.; ROCHA, M. S.; GOMES, C. C. F. Avaliação da Qualidade Protéica da Quinua Real (Chenopodium Quinoa Wild) através de Métodos Biológicos. E-scientia, Belo Horizonte. v.1. n.1., nov. 2008.

ANJO, D. L. C. Alimentos funcionais em angiologia e cirurgia vascular. Jornal Vascular Brasileiro, v. 3, n. 2, p. 145- 154, 2004.

AGÊNCIA NACIONAL DE VIGILÂNCIA SANITÁRIA - ANVISA. Informe Técnico N. 50/2012. Aprova "Teor de sódio dos alimentos 
processados". 2012. Disponível em: https://goo.gl/QBLFkZ Acesso em: 14 de setembro de 2014 .

BOOF, C. C. et al. Desenvolvimento de sorvete de chocolate utilizando fibra de casca de laranja como substituto de gordura. Revista Ciência Rural, Santa Maria - RS, v.43, n.10, p. 1892-1897, 2013.

BRAGA, E. O.; MENDONÇA, L. G. Discussão do uso racional da ração humana, com enfoque para seus principais constituintes: linhaça e quinoa. Perspectivas da Ciência e Tecnologia, Rio de Janeiro, v. 2, n. 1-2, 2010.

BRASIL. Ministério da Saúde. Agência Nacional de Vigilância Sanitária. Legislação Visa Legis. Resolução RDC №12, de 02 de janeiro de 2001. Aprova a "Regulamento Técnico sobre padrões microbiológicos para alimentos". Diário Oficial da União, Brasília, 10 de janeiro de 2001.

BRASIL. Ministério da Saúde. Agência Nacional de Vigilância Sanitária. Legislação Visa Legis. Resolução RDC №3, de 15 de janeiro de 2007. Aprova a "Atribuição de Aditivos e seus Limites Máximos para a Categoria de Alimentos 3: Gelados Comestíveis". Diário Oficial da República Federativa do Brasil. Brasília, 15 de janeiro de 2007.

BRASIL. Ministério da Saúde. Agência Nacional de Vigilância Sanitária. Legislação Visa Legis. Resolução RDC №266, de 22 de setembro de 2005. Aprova o "Padrão de identidade e qualidade de gelados comestíveis". Diário Oficial da República Federativa do Brasil, Brasília, 23 de setembro de 2005.

BRASIL. Ministério da Agricultura, Pecuária e Abastecimento. Instrução Normativa №62 de 26 de agosto de 2003. Oficializa os "Métodos de Analíticos Oficiais para Análises Microbiológicas para controle de Produtos de Origem Animal e Água". Diário Oficial da União, Brasília, 18 de setembro de 2003, Seção 1, p. 14.

CHINELATE, G. C. Et al. Aspectos físico-químicos e microbiológico de gelados comestíveis de leite de búfala adicionados de fibras ali- 
mentares. Revista Brasileira de Agrotecnologia, V. 1, n. 1, p. 0712, jun, 2012.

GALVAO, E.L. et al. Avaliação do potencial antioxidante e extração subcritica do óleo de linhaça. Ciência e Tecnologia de Alimentos, v.28, n.3, p. 551-557, jul. 2008.

INSTITUTO ADOLFO LUTZ. Normas analíticas do Instituto Adolfo Lutz: Métodos Químico e Físico para Análise de Alimentos. 3 ed. São Paulo: Instituto Adolfo Lutz, 2004.

MADRID, A. V.; CENZANO, I.; VICENTE, J.M. Manual de indústria dos alimentos. São Paulo: Livraria Varela, 1996, 599 p.

MARSHALL, R. T.; ARBUCKLE, W.S. Ice cream. 5th ed. New York: International Thomson Publ, 1996.

MARQUES, A.C. Propriedades funcionais da Linhaça (Linum usitatissimum L.) em diferentes condições de preparo e de uso em alimentos. 2008. Dissertação (Mestrado em Ciência e Tecnologia dos Alimentos)- Centro de Ciências Rurais, Universidade Federal de Santa Maria, Rio Grande do Sul, 2008.

MELLO, E. D.; LUFT, V. C.; MEYER, F. Obesidade infantil: como podemos ser eficazes?. Jornal de Pediatria, v.80 , no 3, jan. 2004.

MORRIS, D. H. Linaza: Una recopilación sobre sus efectos en la salud y nutrición. 2007. Disponível em: http://www.flaxcouncil.ca/spanish/pdf/FlxPrmr-R11-Intro_Span.pdf. Acesso em: 15 de setembro de 2014.

NASCIMENTO, L. et al. Sorvete Funcional à base de extrato de Hibisco (Hibiscus sabdariffa L.) adicionado do cálice da flor. 2012. Disponível em: https://goo.gl/c3gz8B Acesso em: 08 set. 2014.

NEUTZLING, M.B. et al. Frequência de consumo de dietas ricas em gordura e pobres em fibra entre adolescentes. Revista Saúde Pública, v.41, n.3, p. 336-342, jun. 2007. 
NEVEZ, C. V. B.; RIBEIRO, D. M. A importância das fibras dietéticas. Programa de Educação Tutorial, Escola de Nutrição. Universidade Federal de Ouro Preto. Murais, 2003.

RAUD, C. Os alimentos funcionais: a nova fronteira da indústria alimentar análise das estratégias da danone e da nestlé no mercado brasileiro de iogurtes. Revista de Sociologia e Política, v. 16, $\mathrm{n}^{\circ}$ 31, 2008.

SANTOS, B.M. Interferência dos ácidos graxos ômega -3 nos lipídeos sanguíneos de ratos submetidos ao exercício físico (NADO). 2006. Dissertação (Mestrado em Nutrição), Universidade Federal de Santa Catarina, Florianópolis, 2006.

SILVA, L. M. R. Et al. Processamento de bolo com farinha de quinoa (chenopodium quinoa willd): estudo de aceitabilidade. Revista Brasileira de Produtos Agroindustriais, Campina Grande, v. 12, n.2, p. 125-132, 2010.

SILVA, N. Relação entre dieta e saúde: o conceito de alimentos funcionais. Engenharia de Alimentos, RPA Editorial, São Paulo, v. 26, p. 40-42, 1999.

SILVA, J. E., LANNES, S.C.S. Effect of different sweetener blends and fat types on ice cream properties. 2011. Disponível 
em: <http://www.scielo.br/scielo.php?script=sci_arttext\&pid=S0101 20612011000100033>. Acesso em: 17 de novembro de 2014.

SOUZA, P.H.M et al. Componentes Funcionais nos Alimentos. Boletim da Sociedade Brasileira de Ciência e Tecnologia em Alimentos, Campinas, v.37, p. 127-135, 2003.

SPEHAR, C.R. Adaptação da Quinoa (Chenopodium quinoa willd.) para incrementar a diversidade agrícola e alimentar no Brasil. Caderno de Ciência \& Tecnologia, 23(1), 41-62, 2006.

TRUCOM. C. A importância da Linhaça na Saúde. São Paulo: Alaude, 2006. 